\title{
A MODEL TO EXPLORE LEARNING PROCESSES IN LEARNER- GENERATED DIGITAL MEDIA (LGDM) ASSIGNMENTS
}

\author{
J. Reyna \\ University of Technology Sydney (AUSTRALIA) \\ jorge.reyna@uts.edu.au
}

\begin{abstract}
Learner-Generated Digital Media (LGDM) as an assessment tool was implemented more than a decade ago in higher education. However, the field is considered under-researched, under-theorised and in the embryonic stage. The theoretical underpinnings to implement LGDM in the classroom use Semiotic Theory, the Self-Explanation effect and the process of internalisation. These instructional theories proposed that learning using LGDM occurs by a relationship between the content, the modality and the construct. When students are asked to produce digital media assignments, they will need to develop a storyboard (content), take in consideration the modality (e.g., audio, images, moving text, animations), and the construct (digital artefact). This approach led to qualitative research to intent to understand how learning happens when using digital media as an assessment tool. This paper proposes a model to explain the learning process when using LGDM in the classroom. The proposed framework has three stages: Learn, Represent and Reinforce. Learn is relate to the preparation of a storyboard. Represent is the stage of multimodal visualisation of content (e.g., images, sound, animation, video), while Reinforce refers to the production of the digital artefact. The model uses the cognitive load theory to explain the learning processes when creating digital media as an assessment tool. Additionally, the model includes self-regulation and motivational factors, and it will allow the use of psychometric tools to test the assumptions. The author hopes this model will help to understand how students learn when using LGDM in the classroom.
\end{abstract}

Keywords: Learner-generated digital media, student-created digital media, user-generated content, authentic assessments, digital media literacies, science education.

This is a preview of the paper to be published at INTED 2019 Proceedings at the end of March 2019.

\section{Reference:}

Reyna, J (2019). A Model to Explore Learning Processes in Learner-Generated Digital Media (LGDM) Assignments. Exploring New Frontiers in Education. The 13th annual International Technology, Education and Development Conference, INTED2019, Valencia (Spain), March 11th-13th. 